\title{
Is self-report sleepiness associated with cognitive performance in temporal lobe epilepsy?
}

\author{
A sonolência auto-relatada está associada ao desempenho cognitivo na epilepsia \\ do lobo temporal?
}

Helena Dresch Vascouto', Maria Emilia Rodrigues de Oliveira Thais', Camila Moreira Osório', Juliana Ben', Lucia Sukys Claudino, ${ }^{1,2}$, Alexandre Ademar Hoeller', Hans J. Markowitsch ${ }^{3}$, Peter Wolf2,4,5, Katia Lin ${ }^{2,4}$, Roger Walz ${ }^{2,4}$

\begin{abstract}
Sleepiness and cognitive impairment are common symptoms observed in patients with epilepsy. We investigate whether self-reported sleepiness is associated with cognitive performance in patients with refractory mesial temporal lobe epilepsy with hippocampal sclerosis (MTLE-HS). Seventy-one consecutive patients with MTLE-HS were evaluated with the Stanford Sleepiness Scale (SSS) before neuropsychological evaluation. Their mean SSS scores were compared with controls. Each cognitive test was compared between patients with (SSS $\geq 3$ ) or without sleepiness (SSS < 3). Imbalances were controlled by regression analysis. Patients reported a significantly higher degree of sleepiness than controls $(p<0.0001)$. After multiple linear regression analysis, only one test (RAVLT total) remained associated with self-reported sleepiness. Conclusion: Self-reported sleepiness was significantly higher in MTLE-HS patients than controls, but did not affect their cognitive performance. If confirmed in other populations, our results may have implications for decision making about sleepiness screening in neuropsychological settings.
\end{abstract}

Keywords: neuropsychological tests; drug resistant epilepsy; clinical decision-making.

\section{RESUMO}

A sonolência e o comprometimento cognitivo são queixas comuns na epilepsia. Investigamos se a sonolência relatada pelo paciente está associada ao desempenho cognitivo na epilepsia do lobo temporal mesial refratária com esclerose do hipocampo (ELTM-EH). 71 pacientes com ELTM-EH foram avaliados pela Escala de Sonolência de Stanford (ESS) antes da avaliação neuropsicológica. A média na ESS foi comparada com a de controles. Cada teste foi comparado entre os pacientes com sonolência (ESS $\geq 3$ ) ou sem sonolência (ESS $<3$ ). Diferenças foram controladas por regressão logística múltipla. Os pacientes relataram uma sonolência maior do que os controles ( $p<0,0001)$. Após a regressão, a sonolência relatada pelos pacientes mostrou-se associada a apenas um teste (RAVLT total). Os pacientes com ELTM-EH referem mais sonolência do que os controles, mas esta não foi associada com a cognição. Se confirmado em outras populações, nossos resultados implicarão na tomada de decisão sobre o impacto da sonolência no contexto neuropsicológico.

Palavras- chave: testes neuropsicológicos; epilepsia resistente a medicamentos; tomada de decisão clínica.

Mesial temporal lobe epilepsy related to hippocampal sclerosis (MTLE-HS) is the most common focal epilepsy, characterized by seizures that are often refractory to antiepileptic drugs (AEDs), as well as being widely referred for surgical treatment ${ }^{1}$. The impact of MTLE-HS conditions on a patient's daily activities and quality of life is extensive, increasing psychiatric disorders, cognitive impairments and sleep problems ${ }^{2,3}$.
Patients with epilepsy usually complain of daytime sleepiness, ${ }^{4}$ which has a serious impact on their quality of life ${ }^{5}$. This symptom reflects a decrease in physiological processes that maintain vigilance ${ }^{6}$ and may arise from a variety of factors present in epilepsy, such as acute effects of daytime and nocturnal seizures ${ }^{3}$, polytherapy or daily dose of $\mathrm{AEDs}^{7}$, coexistence of primary sleep disorders ${ }^{8}$, including abnormalities of sleep microarchitecture ${ }^{9}$ or psychiatric comorbidities ${ }^{10}$.

'Universidade Federal de Santa Catarina, Hospital Universitário, Centro de Neurociências Aplicadas, Florianópolis SC, Brasil;

${ }^{2}$ Universidade Federal de Santa Catarina, Hospital Universitário, Departamento de Clínica Médica, Serviço de Neurologia, Florianópolis SC, Brasil;

${ }^{3}$ University of Bielefeld, Physiological Psychology, Bielefeld, Germany;

«Universidade Federal de Santa Catarina, Departamento de Clínica Médica, Florianópolis SC, Brasil;

${ }^{5}$ Danish Epilepsy Center, Dianalund, Denmark.

Correspondence: Roger Walz; Departamento de Clínica Médica, HU-UFSC; R. Eng. Agronômico Andrei Cristian Ferreira, s/n; $88040-970$ Florianópolis SC, Brasil.Email: rogerwalz@hotmail.com

Conflict of interest: Prof. Dr. Hans J. Markowitsch (Process 406929/2013-0) and Prof. Dr. Peter Wolf (Process 88881.030478/2013-01) are Special Visiting Researchers (PVE) supported by The Science Without Borders Program Project from MEC/MCTI/CAPES/CNPq/FAPs. HDV and CMO received graduate students fellowships from CAPES/CNPq-Brazil. AAH is a post-doctoral fellow supported by PNPD program of CAPES. RW and KL are researcher fellows from CNPq-Brazil.

Support: PRONEX Program (Programa de Núcleos de Excelência - NENASC Project) of FAPESC-CNPq-MS, Santa Catarina Brazil (Grant 56802/2010).

Received 19 February 2018; Received in final form 24 April 2018; Accepted 15 May 2018 
Sleep problems and neuropsychological impairment are intrinsically tied to the epilepsy condition. A decrease in cognitive performance is highly prevalent in patients with MTLE-HS and impacts the domains of memory, language, attention, executive functions and nonverbal processes, according to the side of the sclerosis or surgical resection ${ }^{11,12}$. Although many studies show a relationship between sleep and cognitive performance in different neurological disorders ${ }^{13,14}$, the literature is scarce regarding this issue in epilepsy, especially when sleepiness and low mental activity are common feelings among patients.

Given that clinical neuropsychologists are concerned with the factors associated with cognitive dysfunction, undervaluing the existence of sleep problems at the time of evaluation may affect the interpretation of neuropsychological findings ${ }^{15}$. Recommendations for sleep screening in the neuropsychological context include the examination of subjective sleepiness, with instruments such as the Stanford Sleepiness Scale (SSS), as well as the Epworth Sleepiness Scale, to determine the impact on daily activities ${ }^{15}$. There have been no studies investigating whether a self-reported sleepiness at the time of evaluation may be useful to predict the cognitive performance in patients with epilepsy.

Understanding the association between the degree of sleepiness perceived by the patient and neuropsychological findings may have a daily clinical implication: should patient perception of sleepiness be considered during the interpretation of cognitive performance? Considering the cognitive morbidity that may occur after surgery for MTLE-HS ${ }^{12}$, the use of the perception of sleepiness by the patient as a predictive variable for their cognitive performance may have further implications for decision making in epilepsy surgery.

Our hypothesis was that self-reported sleepiness immediately before the neuropsychological evaluation is negatively associated with cognitive performance in patients with refractory MTLE-HS.

\section{METHODS}

\section{Patients and presurgical evaluation}

Seventy-one consecutive adults diagnosed with refractory MTLE-HS were evaluated between August 2008 and July 2012, at the Centro de Epilepsia de Santa Catarina. Neurologists, neurophysiologists, neurosurgeons, psychiatrists, nurses and neuropsychologists formed the epilepsy surgery team responsible for the patient care. Variables of interest were prospectively collected during hospitalization of patients for their preoperative evaluation using a protocol approved by the Research Ethics Committee Human Research of the Federal University of Santa Catarina (No. 515) and Governador Celso Ramos Hospital (No. 20012/0007). All participants signed an informed consent form and voluntarily agreed to participate in this study.
Drug-resistant epilepsy was defined as the failure to respond to at least two AEDs in appropriate doses for at least 12 months each, with patients having seizures with consciousness impairment. The diagnostic process of MTLE-HS was conducted by a full interview, including clinical history, neurological examination, psychiatric and neuropsychological evaluation, seizure semiology, interictal and ictal videoEEG analysis, MRI (1.5T) and psychosocial assessment ${ }^{2}$. All patients presented with complex focal seizures with or without epigastric, autonomic or psychic auras.

The MRI showed hippocampal sclerosis characterized by a signal increase (FLAIR sequences and T2) and decreased hippocampal volume (FLAIR sequence, T1 or T2). Patients with asymmetric bilateral abnormalities showed the abovementioned findings in one of the hippocampi and atrophy or less evident signal abnormalities in the contralateral hippocampus.

The clinical and demographic characteristics analyzed were: gender, marital status, age, age at epilepsy onset, years of education, hand dominance, work activity, hours of sleep during the night before evaluation, positive history of a precipitant insult, epilepsy duration up to the presurgical assessment, side of mesial temporal abnormality and number of AEDs used.

The use of a single AED or one combined with a benzodiazepine represented the monotherapy patients. Patients who used two or more AEDs, associated or not with benzodiazepines, were classified as being in polytherapy. The AEDs used were: phenytoin, carbamazepine, phenobarbital, lamotrigine, topiramate, oxcarbazepine, and valproic acid. The benzodiazepines used were clobazam or clonazepam, with chronic administration. On the first day of hospitalization, patients maintained the use of their AEDs. The reduction of the drugs was started on the second day, after the evaluation of sleepiness.

For comparisons, the sleepiness levels reported by patients were compared with those reported by healthy controls $(n=36)$. The controls comprised the accompanying persons of patients from other clinics at our university hospital, who were matched for age, gender and education level. The controls were not related to the MTLE-HS patients included in this study.

\section{Sleep evaluation}

The Stanford Sleepiness Scale (SSS) is a well-established instrument for measuring subjective sleepiness for research and clinical purposes, including in patients with epilepsy ${ }^{4,16}$. The instrument was applied immediately before the presurgical neuropsychological evaluation as previously described ${ }^{4}$. Participants were instructed to select one of seven descriptors that best described their current state of sleepiness, ranging from "alert" to "almost asleep". The statements and their scale values are as follows ${ }^{17}$ :

1. Feeling active, vital, alert, or wide awake;

2. Functioning at a high level, but not at peak; able to concentrate; 
3. Relaxed; awake; not at full alertness; responsive;

4. A little foggy, not at peak; let down;

5. Fogginess; beginning to lose interest in remaining awake; slowed down;

6. Sleepiness; prefer to be lying down; fighting sleep; woozy; and

7. Almost in reverie; sleep onset soon; lost struggle to remain awake.

A score $\geq 3$ is associated with a decline in performance that is related to sleepiness ${ }^{4}$. The SSS has sensitivity for sleep deprivation ${ }^{18}$, as well as having a positive correlation with sleep latency and a negative correlation with sleep efficiency and total sleep time ${ }^{19}$.

The "hours of sleep overnight" variable was qualitatively measured by asking patients about their sleeping hours in the night before the cognitive evaluation.

\section{Cognitive tests}

The standardized tests were applied by the neuropsychologist of our team, blinded for all clinical, neurosurgical and laboratory variables previously described. On the first night of hospitalization, the patients were oriented to sleep at 11:00 pm and were awakened at 6:00 am on the morning of the cognitive evaluation (second day of hospitalization). Neuropsychological testing started between 9:00 am and 10:00 am.

The sequence of the tests remained the same in all assessments: Category Fluency ${ }^{20}$, Letters Fluency ${ }^{20}$, Rey Auditory Verbal Learning Test (RAVLT) total and retention ${ }^{20}$, Rey-Osterrieth Complex Figure (ROCF) copy and immediate recall ${ }^{20}$, Block Design ${ }^{21}$, Picture Completion ${ }^{21}$, RAVLT delayed and recognition, ROCF delayed, Logical Memory $1^{\text {st }}$ recall and Logical Memory ${ }^{22}$, Visual Reproduction ${ }^{22}$, Digit Span $^{21}$, Vocabulary ${ }^{21}$, Matrix Reasoning ${ }^{21}$, Logical Memory II, Visual Reproduction II, Similarities ${ }^{21}$, Paired Associates $\mathrm{I}^{22}$, Information ${ }^{21}$, Five-Point Test ${ }^{20}$, Paired Associates II and Boston Naming Test ${ }^{20}$.

\section{Statistical analysis}

Continuous variables were expressed as mean \pm standard deviation (SD) and categorical variables were expressed as frequency and percentage values. The Kolmogorov-Smirnov test was used to analyze the normal distribution of the variables. Categorical variables were analyzed by Chi-square or Fisher's exact test.

We compared the mean SSS scores between patients and controls using the Student's " $t$ " test. Based on the SSS score dichotomy $y^{4,17}$, the Student's " $t$ " test was performed to determine the clinical and demographic differences between patients with sleepiness (SSS score $\geq 3$ ) or without sleepiness (SSS $<3$ ), as well as comparisons with the cognitive tests. In this analysis, we identified the demographic, clinical and cognitive variables associated with sleepiness with a level of significance of $p<0.20$.

To exclude whether the association between the neuropsychological tests (dependent variable) and the SSS score were due to confounding bias related to imbalances in the demographic and clinical variables, a multiple linear regression was done. For this analysis, we included the variables showing the association with the SSS with a $\mathrm{p}<0.20$ level of significance. Considering the clinical and biological plausibility of an association between sleepiness and low cognitive performance, to avoid a type II error, a $\mathrm{p}<0.05$ was considered significant for our analysis. Because we were unable to test the degree of difference between the mean score (and SD) of the cognitive test scores in the analyzed groups that could be considered clinically meaningful for our patients, the difference was chosen based arbitrarily on our previous study ${ }^{23}$. In that study, the decrease in the mean score of MTLE-HS patients (expressed in percentage of decrease from controls), among the cognitive tests showing a statistically significant difference between patients and controls, ranged from $22 \%$ (for RAVLT total) to $49 \%$ (RAVLT $49 \%$ ). In both groups, the SD from the mean ranged from $5 \%$ to $20 \%$ of the mean, depending on the cognitive test analyzed. We considered the lower percentage of change in the mean $(20 \%)$ and the high percentage of change in the SD $(20 \%)$ from that study to calculate the power of analysis. The power of our analysis (1-b) to detect a difference of $20 \%$ in a cognitive test score mean (with an SD of $20 \%$ of mean) between the patients with (SSS score $\geq 3$ ) or without sleepiness (SSS < 3) was 95\%.

\section{RESULTS}

The demographic characteristics and the SSS scores of patients and controls are shown in Table 1. There were no differences between patients and controls regarding gender, hand dominance, age, years of education and hours of night sleep before the SSS evaluation ( $p>0.18$ ). Patients had significantly higher SSS scores than controls $(\mathrm{p}<0.0001)$.

Table 1. Comparison of demographic variables and the Stanford Sleepiness Scale score between patients with refractory MTLE-HS and controls.

\begin{tabular}{|c|c|c|c|}
\hline Variables & $\begin{array}{c}\text { Patients } \\
n=71(\%) \\
n=71(\%)\end{array}$ & $\begin{array}{c}\text { Controls } \\
n=71(\%) \\
n=36(\%)\end{array}$ & $\mathrm{p}$-value \\
\hline \multicolumn{4}{|l|}{ Gender } \\
\hline Female & $37(52.1)$ & $23(63.9)$ & \\
\hline Male & $34(47.9)$ & $13(36.1)$ & 0.25 \\
\hline \multicolumn{4}{|l|}{ Hand dominance } \\
\hline Right & $66(93.0)$ & $32(89)$ & \\
\hline Left & $5(7.0)$ & $4(11)$ & 0.47 \\
\hline Age (years) & $36(11.5)$ & $35.6(12.3)$ & 0.85 \\
\hline Education (years) & $7.3(3.2)$ & $8.2(2.8)$ & 0.18 \\
\hline Hours of sleep overnight & $6(2.0)$ & $6.56(1.7)$ & 0.18 \\
\hline \multicolumn{4}{|l|}{ Stanford sleepiness scale } \\
\hline Overall score & $3.38(1.8)$ & $1.56(0.8)$ & $<0.0001$ \\
\hline Score $\geq 3(\%)$ & $33(46.5)$ & $3(8.3)$ & $<0.0001$ \\
\hline
\end{tabular}


Clinical and demographic characteristics of all patients with MTLE-HS and their reported level of sleepiness are presented in Table 2. Considering all patients, 37 were female (52.1\%), an average age of $36( \pm 11.5)$ years, mean disease duration of $22.7( \pm 10.6)$ years, and mean age at epilepsy onset of $9.6( \pm 9.1)$ years. There was no significant difference in distribution of gender, marital status, work activity, history of initial precipitating injury (brain infection, prolonged seizure, prolonged febrile seizure, moderate or severe head trauma or labor-related injury), side of hippocampal sclerosis on MRI, hand dominance, age, disease duration, or age of epilepsy onset between patients with or without sleepiness ( $p>0.15$ ). Patients with sleepiness (SSS score $\geq 3$ ) showed nonsignificant trends of higher education levels $(\mathrm{p}=0.06)$, chronic use of benzodiazepines $(p=0.10)$ and lamotrigine $(p=0.06)$. Patients

Table 2. The distribution of clinical and demographic variables according to the Stanford Sleepiness Scale (SSS) scores in patients with refractory MTLE-HS.

\begin{tabular}{|c|c|c|c|c|}
\hline \multirow{4}{*}{ Variables } & \multirow{4}{*}{ All patients } & \multicolumn{2}{|c|}{ Sleepiness ${ }^{a}$} & \multirow{4}{*}{$\mathrm{p}$-value } \\
\hline & & No & Yes & \\
\hline & & $n=71(\%)$ & \multirow{2}{*}{$n=45(\%)$} & \\
\hline & & $n=26(\%)$ & & \\
\hline \multicolumn{5}{|l|}{ Gender } \\
\hline Female & $37(52.1)$ & $13(35.1)$ & $24(64.9)$ & \\
\hline Male & $34(47.9)$ & $13(38.2)$ & $21(61.8)$ & 0.79 \\
\hline \multicolumn{5}{|l|}{ Marital status } \\
\hline Single & $34(47.9)$ & $16(47.1)$ & $18(52.9)$ & \\
\hline Married & $26(36.6)$ & $9(34.6)$ & $17(65.4)$ & \\
\hline Divorced or widower & $7(9.9)$ & $1(14.3)$ & $6(85.7)$ & 0.23 \\
\hline \multicolumn{5}{|l|}{ Work activity } \\
\hline Working & $22(31.0)$ & $07(31.8)$ & $15(68.2)$ & \\
\hline Housewife & $13(18.3)$ & $5(38.5)$ & $8(61.5)$ & \\
\hline Unemployed & $14(19.7)$ & $4(28.6)$ & $10(71.4)$ & \\
\hline Health insurance & $22(31.0)$ & $10(45.5)$ & $12(54.5)$ & 0.71 \\
\hline \multicolumn{5}{|c|}{ History of initial precipitating injury } \\
\hline No & $26(36.6)$ & $8(30.8)$ & $18(69.2)$ & \\
\hline Yes & $38(53.5)$ & $16(42.1)$ & $22(57.9)$ & \\
\hline Unknown & $7(9.9)$ & $2(7.7)$ & $5(71.4)$ & 0.58 \\
\hline \multicolumn{5}{|c|}{ Side of hippocampal sclerosis on MRI } \\
\hline Right & $30(42.3)$ & $9(30.0)$ & $21(70.0)$ & \\
\hline Left & $38(53.5)$ & $17(44.7)$ & $21(55.3)$ & \\
\hline Bilateral ${ }^{b}$ & $3(4.2)$ & $0(0)$ & $3(100.0)$ & 0.18 \\
\hline \multicolumn{5}{|l|}{ AEDs Treatment } \\
\hline Monotherapy & $30(42.3)$ & $12(40.0)$ & $18(60.0)$ & \\
\hline Polytherapy & $41(57.8)$ & $14(34.1)$ & $27(65.8)$ & 0.32 \\
\hline \multicolumn{5}{|l|}{ Benzodiazepines } \\
\hline No & $32(45.1)$ & $15(46.9)$ & $17(53.1)$ & \\
\hline Yes & $39(54.0)$ & $11(28.2)$ & $28(71.8)$ & 0.10 \\
\hline \multicolumn{5}{|l|}{ AEDs in use } \\
\hline Carbamazepine & $53(74.6)$ & $17(32.1)$ & $36(67.9)$ & 0.17 \\
\hline Phenytoin & $11(15.5)$ & 7 (63.6) & $4(36.4)$ & 0.04 \\
\hline Phenobarbital & $19(26.8)$ & $5(26.3)$ & $14(73.7)$ & 0.28 \\
\hline Valproic acid & $13(18.3)$ & $3(23.1)$ & $10(76.9)$ & 0.26 \\
\hline Topiramate & $5(7.0)$ & $3(60.0)$ & $2(40.0)$ & 0.26 \\
\hline Lamotrigine & $10(14.1)$ & $1(10.0)$ & $9(90.0)$ & 0.06 \\
\hline Oxcarbazepine & 7 (9.9) & $4(57.1)$ & $3(42.9)$ & 0.23 \\
\hline \multicolumn{5}{|l|}{ Hand Dominance } \\
\hline Right & $66(93.0)$ & $24(36.4)$ & $42(63.6)$ & \\
\hline Left & $5(7.0)$ & $2(40.0)$ & $3(60.0)$ & 0.87 \\
\hline Age (years) & $36(11.5)$ & $35.5(10)$ & $36.3(12.3)$ & 0.80 \\
\hline Education (years) & $7.3(3.2)$ & 6.35 (3.3) & $7.8(3.1)$ & 0.06 \\
\hline Disease duration (years) & $22.7(10.6)$ & $22.7(8.2)$ & $22.7(11.9)$ & 0.98 \\
\hline Epilepsy onset age (years) & $9.6(9.15)$ & $9.1(8.5)$ & $9.9(9.6)$ & 0.73 \\
\hline Hours of sleep overnight ${ }^{c}$ & $6(2.0)$ & $6.9(1.8)$ & $5.5(1.96)$ & 0.02 \\
\hline
\end{tabular}


without sleepiness $(\mathrm{SSS}<3)$ reported longer sleep time before their evaluation $(p=0.02)$. Phenytoin treatment was more frequent in the group without sleepiness $(p=0.04)$. Finally, as seen on MRI, distribution of the side of hippocampal sclerosis according to reported-sleepiness severity was not significant ( $p=0.18$ ).

Comparison of mean scores of each neuropsychological test between patients with or without sleepiness is shown in Table 3. Among all 25 analyzed neuropsychological tests, the RAVLT total ( $p=0.01$ ), RAVLT retention ( $p=0.08)$, RAVLT delayed, ROCF immediate and ROCF delayed were positively associated with greater sleepiness with $\mathrm{p}<0.20$ (Table 3 ).

Table 4 shows that after the multiple linear regression analysis controlling for benzodiazepines and AEDs used (phenytoin, lamotrigine), MRI side of hippocampal sclerosis and education level, only the RAVLT total score variation remained significantly associated with the SSS score variation. The SSS score alone predicted $9 \%\left(r^{2}=0.09, p=0.01\right)$ of the RAVLT total score variation.

\section{DISCUSSION}

Confirming our previous results ${ }^{4}$, the patients with drugresistantMTLE-HS reported a significantly higher level of sleepiness than controls. The non-sleepiness patients (SSS < 3) also had more hours of sleep overnight than the sleepiness patients (1.4 hours longer), which is consistent with the assumption that sleepiness is influenced by sleep duration ${ }^{24}$.

However, contrary to our expectations, we found no association between the degree of perceived sleepiness and cognitive performance in our patients. In addition, a higher cognitive performance in the RAVLT test (high scores) was observed in the sleepiness group. Since there is no clinical or biological plausibility for this association and considering the multiple comparisons that we did, this result may be related to a type I error. Even if we assume that the observed significant association is real, it has no clinical relevance because: i) only one of the 25 neuropsychological tests analyzed could

Table 3. Neuropsychological tests score of MTLE-HS patients according to sleepiness category determined by the Stanford Sleepiness Scale (SSS).

\begin{tabular}{|c|c|c|c|}
\hline \multirow{3}{*}{ Cognitive Tests } & \multicolumn{2}{|c|}{ Sleepiness } & \multirow{3}{*}{ p-value } \\
\hline & No & Yes & \\
\hline & $(n=26)$ & $(n=45)$ & \\
\hline \multicolumn{4}{|l|}{ Verbal memory } \\
\hline Logical Memory $1^{\text {st }}$ & $18.4(7.2)$ & $18.5(7.5)$ & 0.95 \\
\hline Logical Memory I & $29.3(11.2)$ & $29.9(11.1)$ & 0.83 \\
\hline Logical Memory II & $15.2(9.9)$ & $15.2(9.0)$ & 0.99 \\
\hline Paired Associates I & $10.2(6.7)$ & $11.0(7.3)$ & 0.69 \\
\hline Paired Associates II & $2.8(2.5)$ & $3.5(2.6)$ & 0.30 \\
\hline RAVLTTotal & $33(8.5)$ & $38.4(8.9)$ & 0.01 \\
\hline RAVLT Retention & $5.3(3.0)$ & $6.6(2.7)$ & 0.08 \\
\hline RAVLT Delayed & $4.7(3.2)$ & $5.7(3.0)$ & 0.19 \\
\hline RAVLT Recognition & $8.9(3.9)$ & $10.1(6.3)$ & 0.37 \\
\hline \multicolumn{4}{|l|}{ Nonverbal memory } \\
\hline ROCF Immediate & $10.8(4.7)$ & $13.0(6.7)$ & 0.15 \\
\hline ROCF Delayed & $11.6(5.1)$ & $14.3(8.2)$ & 0.13 \\
\hline Visual Reproduction I & $61.2(19.5)$ & $60.7(21.8)$ & 0.93 \\
\hline Visual Reproduction II & $21.9(19.7)$ & $26(23.8)$ & 0.46 \\
\hline \multicolumn{4}{|c|}{ Attention and working memory } \\
\hline Digit Span & $11.6(2.7)$ & $11(3.0)$ & 0.37 \\
\hline \multicolumn{4}{|l|}{ Executive Function } \\
\hline Five-Points Test & $19.1(11.0)$ & $18.6(9.5)$ & 0.85 \\
\hline \multicolumn{4}{|l|}{ Language } \\
\hline Boston Naming Test & $34.1(9.3)$ & $34.3(10.1)$ & 0.93 \\
\hline Letters Fluency & $20.6(6.5)$ & $19.5(8.8)$ & 0.58 \\
\hline Category Fluency & $10.4(3.4)$ & $10.8(4.4)$ & 0.68 \\
\hline Vocabulary & $21.7(7.9)$ & $22.1(7.0)$ & 0.83 \\
\hline Similarities & $14.1(5.8)$ & $15.2(4.5)$ & 0.39 \\
\hline Information & $6(2.8)$ & $6.8(4.5)$ & 0.32 \\
\hline \multicolumn{4}{|c|}{ Visuospatial, visuoperceptual and motor skills } \\
\hline Block Design & $21.1(11.9)$ & $22.8(12.6)$ & 0.58 \\
\hline Matrix Reasoning & $7.8(4.5)$ & $7.7(5.1)$ & 0.91 \\
\hline Picture Completion & $14.3(5.0)$ & $14.22(7.2)$ & 0.94 \\
\hline ROCF Copy & $25.4(6.1)$ & $26.7(6.5)$ & 0.43 \\
\hline
\end{tabular}

RAVLT: Rey Auditory Verbal Learning Test, ROCF: Rey-Osterrieth Complex Figure. 
Table 4. Multiple linear regression analysis showing the independent association between the Stanford Sleepiness Scale (SSS) scores and the neuropsychological tests.

\begin{tabular}{|c|c|c|c|c|}
\hline \multirow{2}{*}{ Cognitive tests and predictors } & \multicolumn{3}{|c|}{ Linear regression coefficients } & \multirow{2}{*}{$p$-value } \\
\hline & $\mathrm{r}$ & $r^{2}$ & $\mathrm{~B}(\mathrm{Cl} 95 \%)$ & \\
\hline RAVLT Total & 0.46 & 0.21 & & 0.001 \\
\hline Side of hippocampal sclerosis on MRI & & & 0.7 (0.1 to 1.4$)$ & 0.03 \\
\hline Education & & & $-2.9(-6.5$ to 0.6$)$ & 0.10 \\
\hline SSS score & & & $1.2(0.004$ to 2.2$)$ & 0.05 \\
\hline SSS score alone & 0.29 & 0.09 & 1.4 (0.3 to 2.6$)$ & 0.01 \\
\hline RAVLT Retention & 0.46 & 0.21 & & 0.001 \\
\hline Side of hippocampal sclerosis on MRIa & & & $-1.5(-2.6$ to -0.36$)$ & 0.01 \\
\hline Education & & & $0.2(-0.02$ to 0.04$)$ & 0.07 \\
\hline SSS score & & & $0.25(-0.1$ to 0.6$)$ & 0.16 \\
\hline ROCF Delayed & 0.42 & 0.18 & & 0.004 \\
\hline Education & & & 0.3 (0.1 to 0.5$)$ & 0.005 \\
\hline SSS & & & $0.28(-0.6$ a 1.2$)$ & 0.86 \\
\hline ROCF Immediate & 0.39 & 0.16 & & 0.004 \\
\hline Education & & & 0.8 (0.3 to 1.2 ) & 0.001 \\
\hline SSS score & & & $-0.2(-1.0$ to 0.6$)$ & 0.63 \\
\hline
\end{tabular}

a For the linear regression analysis the side of hippocampal sclerosis on MRI were classified as: 1 = right hippocampal sclerosis; $2=$ left hippocampal sclerosis: 3 = bilateral hippocampal sclerosis; ${ }^{b}$ SSS score alone predicted $9 \%\left(r^{2}=0.09\right)$ of the RAVLT total score variation; p: significance level. RAVLT: Rey Auditory Verbal Learning Test, ROCF: Rey-Osterrieth Complex Figure.

be predicted by the SSS; ii) the SSS explained only up to $10 \%$ of the RAVLT total score variation.

Few studies in epilepsy have highlighted the relationship between sleep problems and cognitive performance ${ }^{25,26}$. Our study differs from those as we investigated the association of important patient complaints - feelings of sleepiness and cognitive decrease - using a larger sample of MTLE-HS patients tested with various instruments covering different neuropsychological domains. We challenged the general assumption that the degree of sleepiness reported immediately prior to neuropsychological evaluation would interfere with cognitive performance.

As daytime sleepiness is a common feeling among patients with epilepsy $y^{4,9}$, the better clinical choice was to use self-reporting instruments that measured the perception of sleepiness at the time, as recommended by Water and Bucks ${ }^{15}$. One hypothesis for our results is that the subjective approach requires that patients be aware of feeling sleepy and be able to distinguish sleepiness from other factors affecting performance ${ }^{6}$. Depending on the level of somnolence, patients are often not quite accurate in estimating their own level, sometimes falling asleep while rating themselves as fully alert ${ }^{6,27}$. Preliminary studies have also indicated that contextual factors may influence the subjective sleepiness rating, such as the patient's location and level of interest in the activity ${ }^{28}$. Thus, it is perhaps not totally surprising that a self-reported "sleepiness" before a neuropsychological evaluation did not necessarily result in a cognitive impairment.

It is possible that stimulation provided by the test conditions itself may have been sufficient to overcome the sleepiness initially reported by our patients, partly explaining the results found in our samples. A specific decline in cognitive performance may be considered as an indication of the level of sleepiness ${ }^{29}$, but situations presenting interesting, stimulating and engaging tasks are less susceptible to decreased performance, while the presence of a low stimulus or low concentration request tend to increase the somnolence intensity ${ }^{30}$.

The main limitations of our study were the use of only one instrument of self-reported sleepiness and the absence of a validated Portuguese-language version for use in Brazil. The SSS has been recommended in the literature and used in national studies for screening acute subjective sleepiness in neuropsychological settings ${ }^{4,9,15}$, which strongly supported our choice. In addition, other instruments that measure subjective sleepiness at the time, such as the Karolinska Sleepiness Scale, are not widely used for clinical purposes, and even less so in epilepsy research ${ }^{31,32}$. In spite of the limitations, this study is a step towards understanding which sleep complaints may be contributing to the cognitive profile of patients. Future studies may complement our results by adding objective measures of sleepiness and subjective analyses of previous sleep quality.

In summary, subjective sleepiness was significantly higher in MTLE-HS patients than healthy controls but was not associated with their cognitive performance. In consonance with our purposes, the results do not imply that sleepiness does not interfere in cognition, but suggest that the degree of sleepiness perceived by the patient may be disregarded during the interpretation of neuropsychological outcomes. If confirmed in other populations and epilepsy types, our results may have implications for decision making about sleepiness screening in clinical settings. 
1. Engel J Jr. Mesial temporal lobe epilepsy. In: Engel J Jr,, Pedley TA. Epilepsy: a comprehensive textbook. Philadelphia, PA: Lippincott-Raven;1997.

2. Pauli C, Thais ME, Claudino LS, Bicalho MA, Bastos AC, Guarnieri R et al. Predictors of quality of life in patients with refractory mesial temporal lobe epilepsy. Epilepsy \& Behavior. 2012 Oct;25(2):208-13. https://doi.org/10.1016/j.yebeh.2012.06.037

3. Golde EG, Gutter T, Weerd AW. Sleep disturbances in people with epilepsy; prevalence, impact and treatment. Sleep Med Rev. 2011 Dec;15(6):357-68. https://doi.org/10.1016/j.smrv.2011.01.002

4. Pizzatto R, Lin K, Watanabe N, Campiolo G, Bicalho MA, Guarnieri $R$ et al. Excessive sleepiness and sleep patterns in patients with epilepsy: a case-control study. Epilepsy \& Behavior. 2013 Oct;29(1):63-6. https://doi.org/10.1016/j.yebeh.2013.06.029

5. Piperidou C, Karlovasitou A, Triantafyllou N, Terzoudi A, Constantinidis T, Vadikolias K et al. Influence of sleep disturbance on quality of life of patients with epilepsy. Seizure. 2008 Oct;17(7):58894. https://doi.org/10.1016/j.seizure.2008.02.005

6. Guilleminault C, Brooks SN. Excessive daytime sleepiness: a challenge for the practising neurologist. Brain. 2001 Aug;124(Pt 8):1482-91. https://doi.org/10.1093/brain/124.8.1482

7. Zhou JY, Tang XD, Huang LL, Zhong ZQ, Lei F, Zhou D. The acute effects of levetiracetam on nocturnal sleep and daytime sleepiness in patients with partial epilepsy. J Clin Neurosci. 2012 Jul;19(7):95660. https://doi.org/10.1016/j.jocn.2011.09.032

8. Almeida CA, Lins OG, Lins SG, Laurentino S, Valença MM. [Sleep disorders in temporal lobe epilepsy]. Arq Neuropsiquiatr. 2003 Dec;61(4):979-87. Portuguese. https://doi.org/10.1590/S0004-282X2003000600017

9. Scarlatelli-Lima AV, Sukys-Claudino L, Watanabe N, Guarnieri R, Walz R, Lin K. How do people with drug-resistant mesial temporal lobe epilepsy sleep? A clinical and video-EEG with EOG and submental EMG for sleep staging study. eNeurologicalSci. 2016 Jun;4:34-41. https://doi.org/10.1016/j.ensci.2016.06.002

10. Moser D, Pablik E, Aull-Watschinger S, Pataraia E, Wober C, Seidel S. Depressive symptoms predict the quality of sleep in patients with partial epilepsy: a combined retrospective and prospective study. Epilepsy \& Behavior. 2015 June;47:104-10. https://doi.org/10.1016/j.yebeh.2015.04.021

11. Zhao F, Kang H, You L, Rastogi P, Venkatesh D, Chandra M. Neuropsychological deficits in temporal lobe epilepsy: a comprehensive review. Ann Indian Acad Neurol. 2014 Oct;17(4):374-82. https://doi.org/10.4103/0972-2327.144003

12. Sherman EM, Wiebe S, Fay-McClymont TB, Tellez-Zenteno J, Metcalfe A, Hernandez-Ronquillo Let al. Neuropsychological outcomes after epilepsy surgery: systematic review and pooled estimates. Epilepsia. 2011 May;52(5):857-69. https://doi.org/10.1111/j.1528-1167.2011.03022.x

13. Mahmood O, Rapport LJ, Hanks RA, Fichtenberg NL. Neuropsychological performance and sleep disturbance following traumatic brain injury. J Head Trauma Rehabil. 2004 Sep-Oct;19(5):378-90. https://doi.org/10.1097/00001199-200409000-00003

14. Limoges É, Bolduc C, Berthiaume C, Mottron L, Godbout R. Relationship between poor sleep and daytime cognitive performance in young adults with autism. Res Dev Disabil. 2013 Apr;34(4):132235. https://doi.org/10.1016/j.ridd.2013.01.013

15. Waters F, Bucks RS. Neuropsychological effects of sleep loss: implication for neuropsychologists. J Int Neuropsychol Soc. 2011 Jul;17(4):571-86. https://doi.org/10.1017/S1355617711000610
16. Salinsky MC, Oken BS, Binder LM. Assessment of drowsiness in epilepsy patients receiving chronic antiepileptic drug therapy. Epilepsia. 1996 Feb;37(2):181-7. https://doi.org/10.1111/j.1528-1157.1996.tb00010.x17.

17. Hoddes E, Zarcone V, Smythe H, Phillips R, Dement WC. Quantification of sleepiness: a new approach. Psychophysiology. 1973 Jul;10(4):431-6. https://doi.org/10.1111/j.1469-8986.1973.tb00801.x

18. Thomas M, Sing H, Belenky G, Holcomb H, Mayberg H, Dannals R et al. Neural basis of alertness and cognitive performance impairments during sleepiness. I. Effects of $24 \mathrm{~h}$ of sleep deprivation on waking human regional brain activity. J Sleep Res. 2000 Dec;9(4):335-52. https://doi.org/10.1046/j.1365-2869.2000.00225.x

19. Sauter C, Asenbaum S, Popovic R, Bauer H, Lamm C, Klösch G et al. Excessive daytime sleepiness in patients suffering from different levels of obstructive sleep apnoea syndrome. J Sleep Res. 2000 Sep;9(3):293-301. https://doi.org/10.1046/j.1365-2869.2000.00211.x

20. Lezak MD. Neuropsychological assessment. Oxford: Oxford University Press; 2004.

21. Wechsler D. WAIS-III: Wechsler adult intelligence scale: Psychological Corporation San Antonio, TX; 1997.

22. Wechsler D. Wechsler memory scale (WMS-III). San Antonio: Psychological Corporation; 1997.

23. Osório CM, Latini A, Leal RB, Thais MERO, Vascouto HD, Remor AP et al. Neuropsychological functioning and brain energetics of drug resistant mesial temporal lobe epilepsy patients. Epilepsy Research. 2017 Dec;138:26-31. https://doi.org/10.1016/j.eplepsyres.2017.10.009

24. Jewett ME, Dijk DJ, Kronauer RE, Dinges DF. Dose-response relationship between sleep duration and human psychomotor vigilance and subjective alertness. Sleep. 1999 Mar;22(2):171-9. https://doi.org/10.1093/sleep/22.2.171

25. Deak MC, Stickgold R, Pietras AC, Nelson AP, Bubrick EJ. The role of sleep in forgetting in temporal lobe epilepsy: a pilot study. Epilepsy Behav. 2011 Aug;21(4):462-6. https://doi.org/10.1016/j.yebeh.2011.04.061

26. Sarkis RA, Alam J, Pavlova MK, Dworetzky BA, Pennell PB, Stickgold R et al. Sleep-dependent memory consolidation in the epilepsy monitoring unit: a pilot study. Clin Neurophysiol. 2016 Aug;127(8):2785-90. https://doi.org/10.1016/j.clinph.2016.05.275

27. Thorpy MJ, Billiard M, editors. Sleepiness: causes, consequences and treatment. Cambridge University Press; 2011.

28. Sharafkhaneh A, Hirshkowitz M. Contextual factors and perceived self-reported sleepiness: a preliminary report. Sleep Med. 2003 Jul;4(4):327-31. https://doi.org/10.1016/S1389-9457(03)00002-9

29. Pilcher JJ, Huffcutt Al. Effects of sleep deprivation on performance: a meta-analysis. Sleep. 1996 May;19(4):318-26. https://doi. org/10.1093/sleep/19.4.318

30. Cluydts R, De Valck E, Verstraeten E, Theys P. Daytime sleepiness and its evaluation. Sleep Med Rev. 2002 Apr;6(2):83-96. https://doi. org/10.1053/smrv.2002.0191

31. Shahid A, Shen J, Shapiro CM. Measurements of sleepiness and fatigue. J Psychosom Res. 2010 Jul;69(1):81-9. https://doi.org/10.1016/j.jpsychores.2010.04.001

32. Miley AA, Kecklund G, Åkerstedt T. Comparing two versions of the Karolinska Sleepiness Scale (KSS). Sleep Biol Rhythms. 2016;14(3):257-60. https://doi.org/10.1007/s41105-016-0048-8 